\title{
The Mechanistic Basis for Photobiomodulation Therapy of Neuropathic Pain by Near Infrared Laser Light ${ }^{1}$
}

\author{
Vanessa Milanesi Holanda ${ }^{1,2}$ Maria Cristina Chavantes ${ }^{1,3}$ Xingjia $\mathrm{Wu}^{4}$ Juanita J. Anders ${ }^{4}$
}

${ }^{1}$ Post Graduate Program in Biophotonics Applied to the Health Sciences, Universidade Nove de Julho-UNINOVE, São Paulo, SP, Brazil

${ }^{2}$ Center of Neurology and Neurosurgery Associates (CENNA),

Beneficência Portuguesa of São Paulo Hospital, São Paulo, SP, Brazil

${ }^{3}$ Post Graduate Program in Medicine, University Nove de JulhoUNINOVE, São Paulo, SP, Brazil

${ }^{4}$ Uniformed Services University of the Health Sciences, Bethesda, Maryland, USA

Arq Bras Neurocir 2018;37:317-325.
Address for correspondence Vanessa Milanesi Holanda, PhD, Post Graduate Program in Biophotonics Applied to the Health Sciences, Universidade Nove de Julho-UNINOVE, São Paulo, SP, 01504-001 Brazil (e-mail: vanessamila@gmail.com).

\section{Abstract}

\section{Keywords}

- Dorsal Root Ganglion

- Fluence Rate

- Laser Irradiation

- Photoneuromodulation

- Transient Neuronal Injury
Background and Objective Various irradiances have been reported to be beneficial for the treatment of neuropathic pain with near infrared light. However, the mechanistic basis for the beneficial outcomes may vary based on the level of irradiance or fluence rate used. Using in vivo and in vitro experimental models, this study determined the mechanistic basis of photobiomodulation therapy (PBMT) for the treatment of neuropathic pain using a high irradiance.

Study Design/Materials and Methods In vitro experiments: Cultured, rat DRG were randomly assigned to control or laser treatment (LT) groups with different irradiation times $(2,5,30,60$ or $120 \mathrm{~s})$. The laser parameters were: output power $=960 \mathrm{~mW}$, irradiance $=300 \mathrm{~mW} / \mathrm{cm} 2,808 \mathrm{~nm}$ wavelength and spot size $=3 \mathrm{~cm}$ diameter $/$ area $=7.07 \mathrm{~cm} 2$, with different fluences according to irradiation times. Mitochondrial metabolic activity was measured with the MTS assay. The DRG neurons were immunostained using a primary antibody to $\beta$-Tubulin III. In vivo experiments: spared nerve injury surgery (SNI), an animal model of persistent peripheral neuropathic pain, was used. The injured rats were randomly divided into three groups $(n=5) .1)$ Control: SNI without LT, 2) Short term: SNI with LT on day 7 and euthanized on day 7, 3) Long term: SNI with LT on day 7 and euthanized on day 22. An $808 \mathrm{~nm}$ wavelength laser was used for all treatment groups. Treatment was performed once on Day 7 post-surgery. The transcutaneous treatment parameters were: output power: $10 \mathrm{~W}$, fluence rate: $270 \mathrm{~mW} / \mathrm{cm} 2$, treatment time: $120 \mathrm{~s}$. The laser probe was moved along the course of the sciatic/sural nerve during the treatment. Within 1 hour of irradiation, behavior tests were performed to assess its immediate effect on sensory allodynia and hyperalgesia caused by SNI.

Results In vitro experiments: Mitochondrial metabolism was significantly lower compared with controls for all LT groups. Varicosities and undulations formed in
(C) 2017 Wiley Periodicals, Inc. Originally published as Holanda VM, Chavantes MC, Wu X, Anders JJ. The Mechanistic Basis for Photobiomodulation Therapy of Neuropathic Pain by Near Infrared Laser Light. Lasers Surg Med. 2017; doi https://doi.org/10.1002/ lsm.22628.
Copyright (c) 2018 by Thieme Revinter Publicações Ltda, Rio de Janeiro, Brazil 
neurites of DRG neurons with a cell body diameter $30 \mu \mathrm{m}$ or less. In neurites of DRG neurons with a cell body diameter of greater than $30 \mu \mathrm{m}$, varicosities formed only in the 120 s group. In vivo experiments: For heat hyperalgesia, there was a statistically significant reduction in sensitivity to the heat stimulus compared with the measurements done on day 7 prior to LT. A decrease in the sensitivity to the heat stimulus was found in the LT groups compared with the control group on day 15 and 21. For cold allodynia and mechanical hyperalgesia, a significant decrease in sensitivity to cold and pin prick was found within 1 hour after $L T$. Sensitivity to these stimuli returned to the control levels after 5 days post-L T. No significant difference was found in mechanical allodynia between control and L T groups for all time points examined.

Conclusion These in vitro and in vivo studies indicate that treatment with an irradiance/fluence rate at $270 \mathrm{~m} \mathrm{~W} / \mathrm{cm} 2$ or higher at the level of the nerve can rapidly block pain transmission. A combination therapy is proposed to treat neuropathic pain with initial high irradiance/fluence rates for fast pain relief, followed by low irradiance/ fluence rates for prolonged pain relief by altering chronic inflammation.

\section{Introduction}

Neuropathic pain is a common, debilitating disorder with a complex etiology. ${ }^{1}$ Although several pharmacologic agents have been used to treat neuropathic pain, reported outcomes have been poor with less than half of the patients reporting satisfactory relief of their symptoms. ${ }^{2}$ Photobiomodulation therapy (PBMT), previously referred to as low level laser therapy, has been used to decrease various types of neuropathic pain in preclinical animal models and in randomized controlled clinicai trials. PBMT has been reported to reduce pain and improve function in compressive neuropathies such as carpal tunnel syndrome. ${ }^{3}$ Also, systematic reviews and meta-analysis of randomized controlled trials found that PBMT reduced acute and chronic neck pain. ${ }^{4,5}$

Since PBMT can act in part by causing an anti-inflammatory effect in the target tissue, ${ }^{6-9}$ it has promise as an effective treatment for neuropathic pain associated with inflammation. Considering the involvement of the central nervous system in pain, it is important to note that photobiomodulation (PBM) can alter microglial phenotypes: from pro-inflammatory to anti-inflammatory across the Ml/M2 spectrum in a dose-dependent manner. ${ }^{10}$ Several pre-clinical animal studies investigated the effect of PBMT on inflammatory markers in neuropathic pain models. Hsieh and colleagues (2012) reported a decrease in pro-inflammatory markers (tumor necrosis factor (TNF), interleukin $1 \beta$ (IL-1 $\beta$ ), and hypoxia-inducible factor $1-\alpha$ (HIF-1 a)) at a chronic constriction injury site in the rat sciatic nerve in PBMT treated animals compared with non-treated-injured animals. ${ }^{11}$ Cidral-Filho et al. (2013), using a mouse sciatic nerve crush model, reported that PBMT reduced mechanical hypersensitivity and decrease spinal cord and sciatic nerve levels of TNFa. ${ }^{12}$ Recently, our laboratory reported that PBMT effectively reduced mechanical hypersensitivity in a spared nerve injury preclinical model of neuropathic pain and modulated macrophage/microglial activation to an antiinflammatory phenotype. ${ }^{13}$ Both studies provide evidence that PBMT is effective for treating neuropathic pain by altering the inflammatory response. In these studies, the fluences (energy densities) were $9 \mathrm{~J} / \mathrm{cm} 2,{ }^{11} 2.5 \mathrm{~J} / \mathrm{cm} 2,{ }^{12}$ and $8 \mathrm{~J} / \mathrm{cm} 2^{13}$ and the irradiances (power densities) were 150 $\mathrm{mW} / \mathrm{cm} 2$ at the skin surface, ${ }^{11} 80 \mathrm{~mW} / \mathrm{cm} 2$ at the skin surface $^{12}$ and $43.25 \mathrm{~mW} / \mathrm{cm} 2$ at the target tissue. ${ }^{13}$

In contrast, the pioneering work of Dr. Chow et al. on the clinical efficacy of PBMT for neck pain ${ }^{14}$ and the mechanistic basis of pain suppression ${ }^{15}$ used irradiances of $670 \mathrm{~mW} / \mathrm{cm} 2$ at the skin surface for the clinical study ${ }^{14}$ and $300 \mathrm{~mW} / \mathrm{cm} 2$ at the cell surface for the in vitro experiments ${ }^{15}$ at a wavelength of $830 \mathrm{~nm}$. A systematic review on the inhibitory effects of laser irradiation on peripheral mammalian nerves and analgesic effects examined 44 studies: 18 human studies and 26 animal studies. ${ }^{16}$ Although inconsistently reported, irradiances that suppressed conduction velocity and/or reduced the amplitude of the action potentials ranged from $300 \mathrm{~mW} / \mathrm{cm} 2$ to $1.73 \mathrm{~W} / \mathrm{cm} 2$ in the human studies. One important conclusion of this systematic review was that the inhibition of nerve conduction requires comparatively high therapeutic doses. ${ }^{16}$

Recently, we completed a pilot, clinical study for treatment of low back pain that compared three treatment modalities: lidocaine injection, radiofrequency, or PBMT with $808 \mathrm{~nm}$ wavelength light with high irradiance and fluence (measured at the tip of the fiber optic). The data showed that PBMT applied bilaterally to the dorsal root ganglia (DRG) of the second lumbar spinal nerves decreased low back pain within 5 minutes which was comparable to lidocaine injection. ${ }^{17}$ These results lead to the development of this combined in vitro and pre-clinical animal study to better understand the mechanistic basis underlying the photoneuromodulation of the neuropathic low back pain at high irradiances. The data from this study and our previous experiments on nerve regeneration and pain suppression serve as the basis for discussion of mechanisms of action for pain suppression based on irradiance. 


\section{Methods}

\section{In Vitro Experiments}

\section{Cell Culture}

Primary rat dorsal root ganglion neurons (Lonza Walkersville, Inc. Walkersville, MD) were seeded in Poly-D-Lysine/ Laminin $(30 \mu \mathrm{g} / \mathrm{mL}$ poly-D-lysine and $2 \mu \mathrm{g} / \mathrm{mL}$ laminin) coated 4-well chamber slides with a seeding density of $2 \times 104$ cell/well in primary neural growth medium (PNGM) according to manufacturer's instructions. The cells were incubated at $37^{\circ} \mathrm{C}, 5 \% \mathrm{CO2}$ for 48 hours.

\section{Laser Irradiation}

The laser device used for irradiation of the cells was a CW, $808 \mathrm{~nm}$ wavelength diode laser with adjustable output power up to $2 \mathrm{~W}$. Based on our previous clinicai pilot study on low back pain in which a high irradiance was delivered to the $\mathrm{DRG}^{17}$ and a previous in vitro study on DRG neurons, in which $300 \mathrm{~m} \mathrm{~W} / \mathrm{cm} 2$ at different treatment times caused varicosity formation and slowing of nerve conduction in the small DRG neurons, ${ }^{15}$ an irradiance of $300 \mathrm{~mW} / \mathrm{cm} 2$ was used in this study. Measurements were made to determine the output power that was needed to deliver $300 \mathrm{~m} \mathrm{~W} / \mathrm{cm} 2$ to the cells (LabMaster Ultima power meter with LM-3 HTD sensor, Coherent, Inc., Santa Clara, CA). After incubating for 48 hours, the cultures were randomly assigned to either control or laser treatment (LT) groups (irradiation times $=2$, $5,30,60$ or $120 \mathrm{~s}$ ). The laser parameters were: output power $=960 \mathrm{~mW}$, irradiance $=300 \mathrm{~mW} / \mathrm{cm} 2,808 \mathrm{~nm}$ wavelength and spot size: $3 \mathrm{~cm}$ diameter $/$ area $=7,07 \mathrm{~cm} 2$.

\section{MTS Assay}

MTS assay (Promega, Madison, WI) was used to measure the metabolic activity in the living cells. This assay is based on the reduction of tetrazolium salts into formazan, which can be measured colorimetrically. The conversion is presumably accomplished by reductase enzymes in mitochondria. Forty minutes after laser irradiation, MTS solution was added to each well. Forty minutes was chosen based on reports in the literature and previous work in our laboratory that identified that the peak time post-irradiation for change in mitochondrial metabolism was 40 minutes. ${ }^{18}$ After incubation for 1.5 hour at $37^{\circ} \mathrm{C}$, the supernatant was read for absorbance at $485 \mathrm{~nm}$ using FLUOstar OPTIMA plate reader (BMG Labtech Inc. Cary, NC). Blank: controls were medium alone with MTS solution. Each light parameter setting was measured in triplicate. This experiment has been repeated twice and the data were combined.

\section{Immunocytochemistry of P-Tubulin III}

Cells were fixed with $4 \%$ paraformaldehyde and then blocked in PBS with $10 \%$ goat serum and $0.1 \%$ Triton for 30 min at roam temperature (RT). Cells were then incubated with Mouse anti$\beta$-Tubulin III antibody (1:75 in PBS with 1\% goat serum, Sigma, St. Louis, MO) for 1 hour at RT, followed by incubation with secondary antibody (AlexaFluor488 Goat anti-mouse IgG, $2.5 \mu \mathrm{g} / \mathrm{ml}$ in PBS with $1 \%$ goat serum, Life Technologies, Grand Island, NY) for 30 minute at RT. After washing with PBS, samples were cover slipped with Vectashield mounting medium with DAPI (Vectors Laboratories, Inc. Burlingame, CA) and sealed with nail polish. The cells were photographed digitally using an Olympus BX43 fluorescence microscope equipped with an Olympus DP72 microscope digital camera (Olympus Imaging America Inc. Center Valley, PA). The diameters of cell body were measured using Olympus Cel1Sens software (Olympus Imaging America Inc. Center Valley, PA). A minimum of 100 cells from each treatment and control groups was measured.

\section{In Vivo Experiments}

\section{Animals}

The animal use protocol (APG-14-808) was reviewed and approved by the Uniformed Services University of the Health Sciences (USUHS) Institutional Animal Use Committee. Sixteen male Sprague-Dawley (SD) rats (201-225 g, Charles River Laboratories International, Inc., Wilmington, MA) were used in this study. One rat was used for power penetration measurements and the other 15 rats were divided into three groups $(n=5)$ : 1) Control: surgery without LT, 2) Short term: surgery with LTon day 7 and euthanized on the same day after behavior test, 3) Long term: surgery with LT on day 7 and euthanized on day 22. Animals were housed 2 per cage, under a 12-hour light/ dark cycle, with access to food and water ad libitum.

\section{Power Penetration Measurement}

One male SD rat was anesthetized for light penetration measurement. The fluence rate was measured by a near infrared detector which was designed and built by B\&W Tek Inc. (Newark, DE). A small photo sensor $(2.0 \mathrm{~mm} \times 2.5 \mathrm{~mm})$ was sealed in a glass tube. The output voltage of this sensor was calibrated such that a reading of $1 \mathrm{mV}$ represented $1 \mathrm{~m} \mathrm{~W} / \mathrm{cm} 2$ The sensor was placed below the lumbar 4 , and lumbar 5 DRGs. An 808 nm wavelength laser (Model BWF5-808-20, B\&W Tek Inc, Newark, DE), connected with a probe that had a rolling ball with an irradiation diameter of $4 \mathrm{~cm}$, was placed in direct contact with the skin surface. Light penetration was measured for output powers of 3, 5 and $10 \mathrm{~W}$ (which was the maximum output power of this laser). The probe was moved until the highest reading was identified and recorded.

\section{Surgery}

Spared nerve injury (SNI) surgery, an animal model for peripheral neuropathic pain, was performed on all rats. ${ }^{19}$ Briefly, rats were anesthetized with isoflurane (5\% for induction and $0.5-3 \%$ for maintenance). An incision was made on the lateral left thigh and the bicep femoris was separated to expose the sciatic nerve and its branches. The common peroneal and tibial nerves were tight-ligated and 3-4 mm of each nerve (distal to the ligation) were cut and removed. Great care was taken to avoid any contact with the intact sural nerve. The muscle and skin were then sutured in two layers.

\section{Laser Irradiation}

An $808 \mathrm{~nm}$ wavelength laser (Model BWF5-808-20, B\&W Tek Inc, Newark, DE) was used for both the long and short term treatment groups. Transcutaneous laser treatment was 


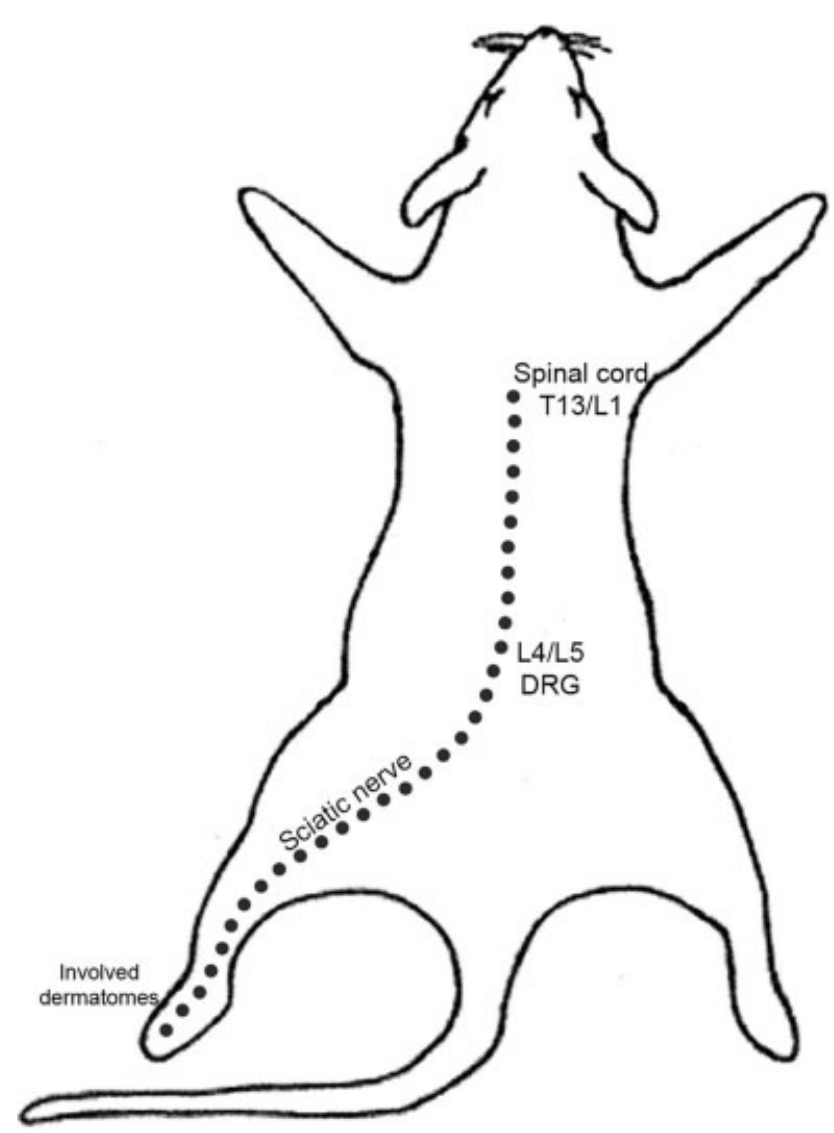

Fig. 1 Illustration of in vivo laser scanning pathway and primary targets.

performed once on Day 7 post-surgery with parameters: output power: $10 \mathrm{~W}$, treatment time: $120 \mathrm{~s}$. The probe had a small massage ball (circular area with a diameter of $4 \mathrm{~cm}$ ) and was scanned along the nerve track during the two minutes of treatment from the thoracic 13 and lumbar 1 (T13/Ll) spinal cord level to the lumbar 4 and 5 (L4/L5) DRG, the sciatic nerve, the sural nerve, and the involved dermatomes on the lateral plantar surface of the hind paw (- Fig. 1). The irradiance rate was $270 \mathrm{~m} \mathrm{~W} / \mathrm{cm} 2$ at the DRG L4/L5 region according to the power penetration measurements. The rats were lightly anesthetized with isoflurane for the laser treatment. The rats in the control group were handled in exactly the same manner as the irradiated rats but the laser was off. Within 1 hour of treatment, behavior tests were performed to assess the immediate effects of the laser treatment on sensory allodynia and hyperalgesia caused by the SNI model. ${ }^{20}$

\section{Behavior Tests}

Behavior tests included Heat Hyperalgesia, Cold Allodynia, Mechanical Hyperalgesia (Pin Prick) and Mechanical Allodynia (Electronic Von Frey). The animals were placed in an inverted plastic box on an elevated metal grid to allow for stimulation on the lateral plantar surface of the hind paw. Tests were performed before surgery (baseline), day 7 before LT and 1 hour after LT and on day 10,12,15, 18 and 21. The behavior tests done on day 7 post-irradiation were done at 1 hour after PBMT because the animals had been anesthetized and needed this amount of time to completely wake up. The behavior data from day 7 before and after $\mathrm{L} \mathrm{T}$ represents the immediate effects from the laser irradiation. For long term effects, data from long term group were compared with the control group.

\section{Heat Hyperalgesia}

The test used to measure heat 1) hyperalgesia was modified from Hargreaves et al. ${ }^{20}$ The thermal stimulation was generated by a beam of radiant heat using an $808 \mathrm{~nm}$ wavelength laser with a $2 \mathrm{~W}$ output power and $3 \mathrm{~mm}$ diameter. Animals were placed on the elevated grid and acclimated for 5 minute. The laser beam was positioned on the lateral plantar surface of the hind paw which is the region innervated by the intact sural nerve. The time when the hind paw was briskly withdrawn was recorded with a maximum cut-off time of 10s. Both left (injured) and right (uninjured) sides were tested. The ratio was calculated as response time of left side divided by the response time of the right site. Rats were tested three times with a 5minute interval between each test.

\section{Cold Allodynia}

After 5 minute of acclimation on the elevated grid, cold acetone $\left(20 \mu \mathrm{l},-20^{\circ} \mathrm{C}\right)$ was sprayed on the lateral plantar surface of the hind paw. The duration of the withdrawal response to the cold stimulation was recorded and graded in 5 levels: 0 , no visible response; 1 , startle response without paw withdrawal; 2, clear withdrawal of the paw; 3, prolonged withdrawal (2-30s) often combined with flinching and licking of the paw; and 4, prolonged, repetitive withdrawal (30s) and/or vocalization.

\section{Mechanical Hyperalgesia (Pin Prick)}

Pin prick test was performed using a safety pin after 5 minute acclimation of the elevated grid. ${ }^{13} \mathrm{~A}$ brief stimulation was applied to the lateral part of the plantar surface of the hind paw. The duration of paw withdrawal was recorded. An arbitrary minimal time of $0.5 \mathrm{~s}$ was used as normal response time. The maximum cut-off time was $15 \mathrm{~s}$.

\section{Mechanical Allodynia (Electronic Von Frey)}

SNI animals developed hypersensitivity to mechanical stimulation, which was measured using an electronic von Frey (Bioseb, Chaville, France) device. ${ }^{21,22}$ The hand-held force transducer of the device can generate force from 0-500 $\mathrm{g}$ in $0.1 \mathrm{~g}$ intervals. After an acclimation period of 15 minute, the plastic tip of the transducer was applied perpendicularly to the lateral plantar surface of the hind paw. The lowest force at which a brisk withdraw of the hind paw was recorded. The test was repeated three times with a 5-minute interval between each measurement, and the mean of the three measurements was computed for analysis.

\section{Statistical Analysis for In Vivo and In Vitro Experiments}

For the MTS assays, one way ANOVA lith Tukey's multiple comparisons test were used to compare the effects between groups. For behavior data, results were presented as mean + standard error of the mean (SEM). Unpaired two-tailed $t$ - 
test was used to compare the immediate effects between before and after irradiation. For longterm behavior data, Two-way ANOVA with Sidak's multiple comparisons test was used to compare control and long-term LT groups. Family-wise significance and confidence levels were set at 0.05 (95\% confidence interval).

\section{Results}

\section{In Vitro Experiments}

All the LT groups with treatment times of 2, 5, 30, 60 and 120s had statistically significant lower mitochondrial metabolism compared with controls (-Fig. 2). There was no difference in the metabolic inhibition for all irradiation times tested. This finding is due to the fact that 40 minutes post irradiation was previously identified as the peak time post-irradiation for change in mitochondrial metabolism. ${ }^{18}$

In all LT groups, varicosities and undulations were present in neurites of DRG neurons with a cell body diameter of 30 $\mu \mathrm{m}$ or less compared with the control group ( - Fig. 3 ). These neurons are associated with $C$ and AS fiber types which in vivo are the unmyelinated and lightly myelinated axons conveying pain and temperature sensory information. The neurites of DRG neurons with a cell body diameter $\geq 30 \mu \mathrm{m}$ began to form varicosities only in the 120 s group (-Fig. 3 ). Neurons of this size are associated with $A \alpha$ and $A \beta$ fiber types, which in vivo are myelinated and convey proprioceptive, two-point tactile and vibration sensory information. It is critical to remember that in our culture model there are no Schwann cells and therefore no myelination of the neurites. Therefore, the response of the neurons to the laser irradiation is not related to the degree of myelination.

\section{In Vivo Experiments}

Power Measurement

For an output power of 3, 5 and $10 \mathrm{~W}$, the fluence rate was measured as $80 \mathrm{~mW} / \mathrm{cm} 2,165 \mathrm{~mW} / \mathrm{cm} 2$ and $270 \mathrm{~mW} / \mathrm{cm} 2$,

\section{MTS assay}

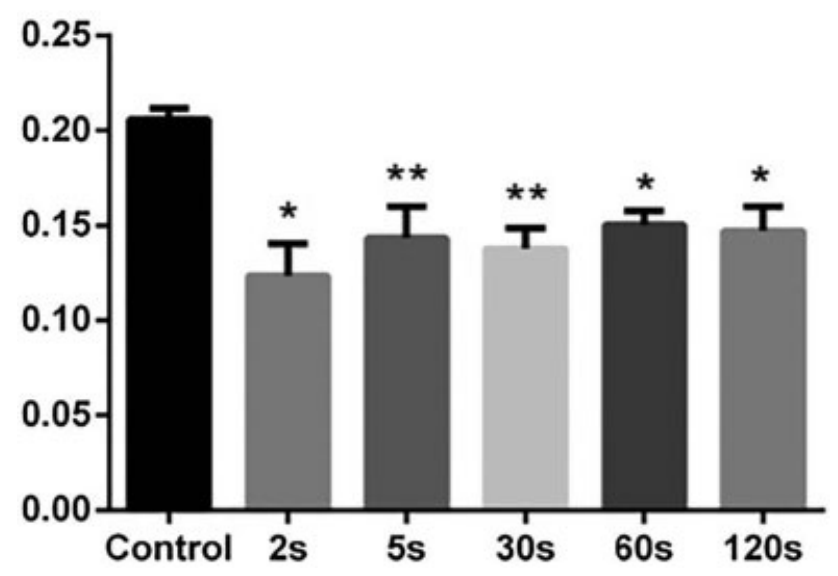

Fig. 2 Statistically significant reduction of mitochondrial metabolism as measured by MTS assay. ${ }^{*} \mathrm{p}<0.005,{ }^{* *} \mathrm{p}<0.01$ compared with control group. respectively. The fluence rate of $270 \mathrm{~m} \mathrm{~W} / \mathrm{cm} 2$ was the closest power density to the target $300 \mathrm{~m} \mathrm{~W} / \mathrm{cm} 2$ which was used in the in vitro experiments. Therefore, an output power of $10 \mathrm{~W}$ was chosen for in vivo laser irradiation experiments.

\section{Behavior Tests}

For all hyperalgesia and allodynia behavior tests done, there was no significant difference found between control and experimental groups on day 7 postsurgery prior to the laser treatments. On day 7 within 1 hour after irradiation, the involved area of the lateral plantar surface of the hind paw was tested.

For heat hyperalgesia, there was a statistically significant decrease in sensitivity to the heat stimulus $(p<0.05)$ compared with the measurements done on day 7 prior to the laser treatment ( - Fig. 4a) within one hour after irradiation. For the long term measurements, a decrease in the sensitivity to the heat stimulus was found in the LT group compared with the control group $(p<0.05)$ on day 15 and day 21 ( - Fig. 4b). The lack of a significant difference on day 12 and day 18 was due to the greater variability in the standard error of the means. A greater number of animals in each group would likely result in decreased heat hyperalgesia for all time points.

For cold allodynia, a significant decrease in sensitivity to the cold stimulus was found within 1 hour post-LT ( $p<0.001$, -Fig. 5a). The sensitivity to the cold stimulus returned to the control levels after 5 days post-LT (Day 12) (-Fig. 5b).

For mechanical hyperalgesia, a significant decrease in sensitivity to the pin prick stimulus $(p<0.05)$ occurred within 1 hour after LT ( $\mathbf{- F i g . 6 a )}$ ) and also returned to the control level after 5 days post-LT on Day 12 (-Fig. 6b).

For mechanical allodynia using electronic Von Frey test, no significant difference was found (-Fig. 7a and $-\mathbf{7 b}$ ) between control and LT groups for all time points examined post-laser treatment.

\section{Discussion}

In the present study, in vitro and pre-clinical animal experiments explored the mechanistic basis underlying photoneuromodulation of neuropathic pain at an irradiance of 300 and $270 \mathrm{~mW} / \mathrm{cm} 2$. Chow et al. (2007) reported that laser irradiation using a CW $830 \mathrm{~nm}$ wavelength laser with an irradiance of $300 \mathrm{~m} \mathrm{~W} / \mathrm{cm} 2$ induced axonal varicosities in DRG small and medium neurons which represent the type $C$ and Ao fibers related to pain, temperature and light touch perception. ${ }^{15}$ Chow et al. ${ }^{11}$ hypothesized that the laser irradiation blocks nociceptor-specific neurons by microtubule disruption with varicosity formation as the key morphological feature. With our treatment parameters, varicosities and undulations formed in in vitro neurites of DRG neurons with a diameter of $30 \mu \mathrm{m}$ or less at all irradiation times examined and in neurites of DRG neurons with a diameter greater than $30 \mu \mathrm{m}$ in the 120 s irradiation group in contrast to Chow's study. These large neurons represent the $A a$ and $A \beta$ large myelinated neurons related to the perception of proprioception, vibration and two point tactile discrimination. 

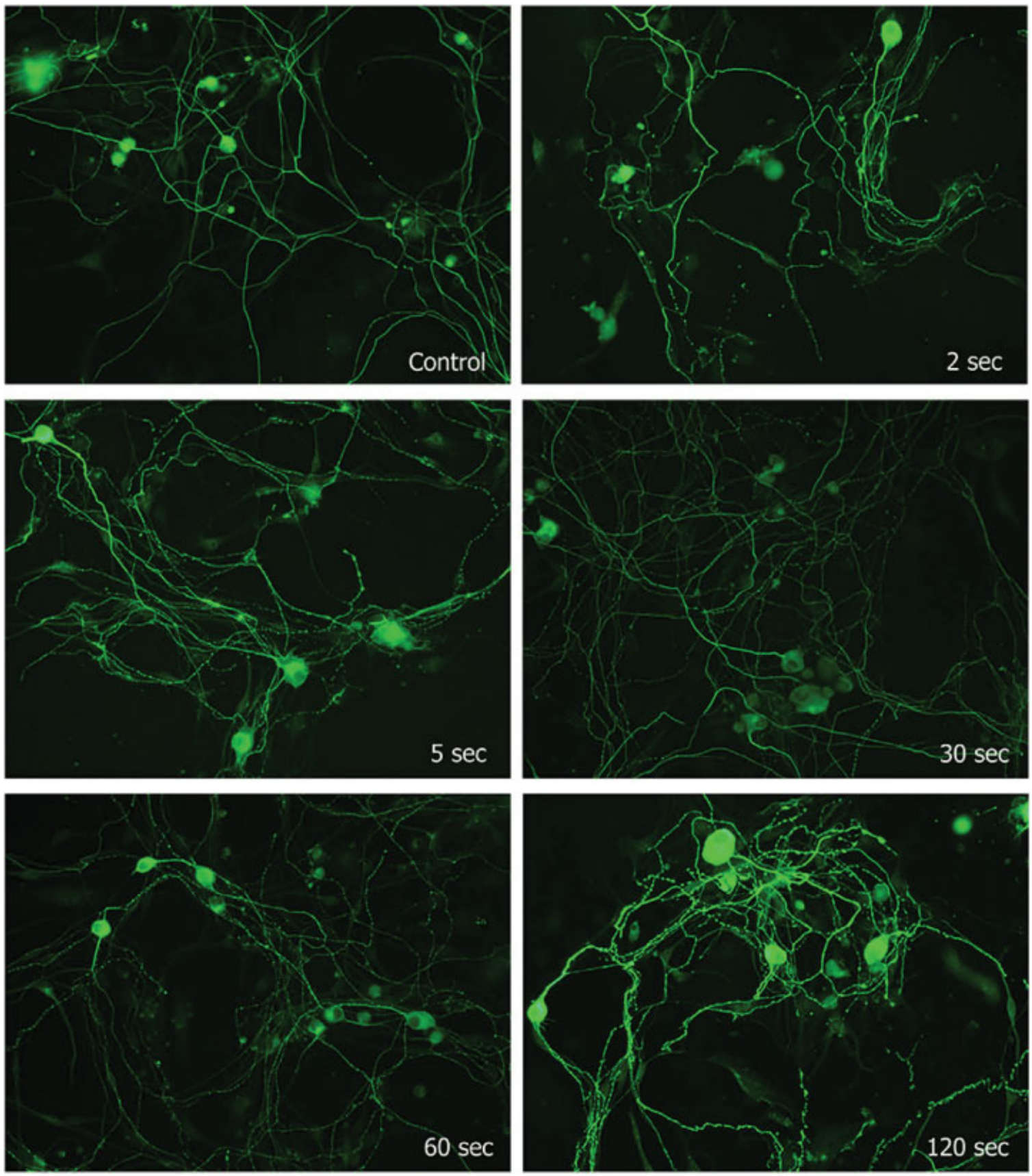

Fig. 3 Photomicrographs of control and L T DRG neurons immune-labeled with $\beta$-Tubulin III. Varicosities and undulations were present in L T groups.

Early varicosity formation in axons have been associated with injury of the central and peripheral nervous systems $\mathrm{s}^{23-25}$ and many neurodegenerative diseases. ${ }^{26,27}$ Interestingly, many reports suggest that varicosity formations in dendrites are neuroprotective (see the recent publication by Liebert for discussion). ${ }^{28}$ Besides laser irradiation, several chemical and physical agents including anesthetics have been reported to cause varicosity formation in neurons of various sizes..$^{29-31}$ Varicosities form when there is breakage of the microtubules. ${ }^{23}$ This cytoskeletal disruption affects axonal flow and mitochondrial function, ${ }^{15,28}$ impairs nerve conduction ${ }^{32}$ and signal transduction. ${ }^{33}$ In the present study, mitochondrial metabolism measured using the MTS assay was significantly lower for all irradiated groups compared with controls. These data were based on the addition of the MTS solution at 40 minutes postirradiation which has been previously identified as the peak time for change in mitochondrial metabolism. ${ }^{18}$ Previously, Chow et al (2007) examined mitochondrial membrane potential in DRG neurons treated with $830 \mathrm{~nm}$ wavelength light with an irradiance of $300 \mathrm{~mW} / \mathrm{cm} 2$ for 30 second. A statistically significant decrease in the mitochondrial membrane potential was found by 5 minute post-irradiation and progressed over the total 30 minutes post-irradiation time. ${ }^{15} \ln$ preliminary studies to determine a maximal irradiance that could block pain transmission and not cause permanent damage, the effect of $600 \mathrm{~m} \mathrm{~W} / \mathrm{cm} 2$ at irradiation times of $60 \mathrm{~s}$ or $120 \mathrm{~s}$ was 
Heat Hyperalgesia (immediately after LT)

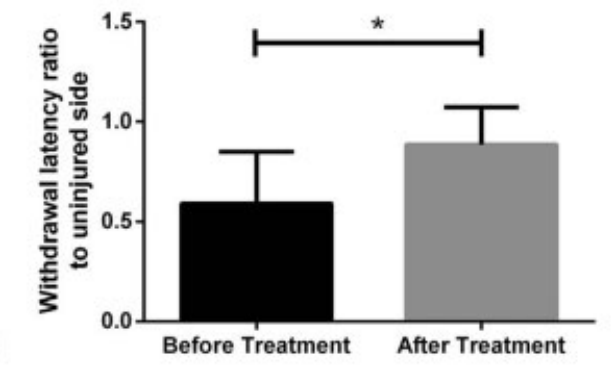

Heat Hyperalgesia (long term)

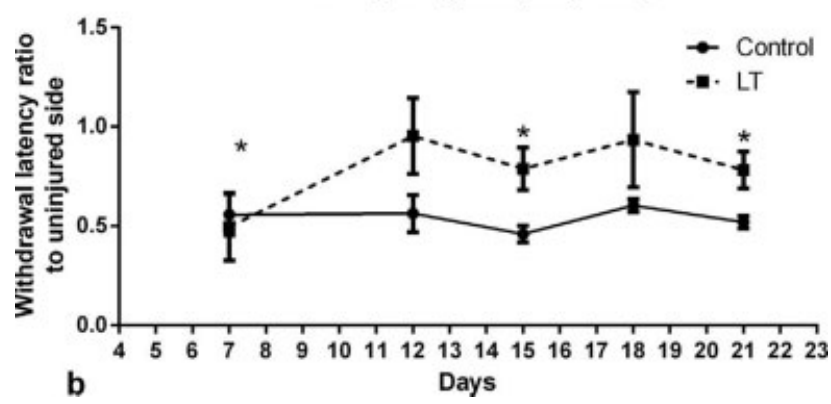

Fig. 4 Heat hyperalgesia. There was a decrease in sensitivity to the heat stimulus $(p<0.05)$ within one hour after irradiation

( - Figure 4a). For the long term, a decrease was found in the LT group $(p<0.05)$ on day 15 and 21 (-Figure $\mathbf{4 b}$ ).

Cold Allodynia (immediately after LT)

a

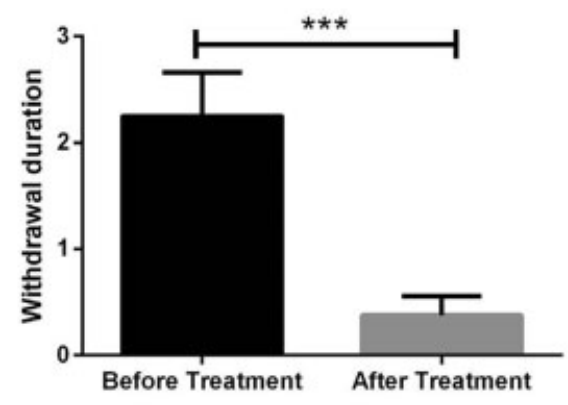

Cold Allodynia (long term)

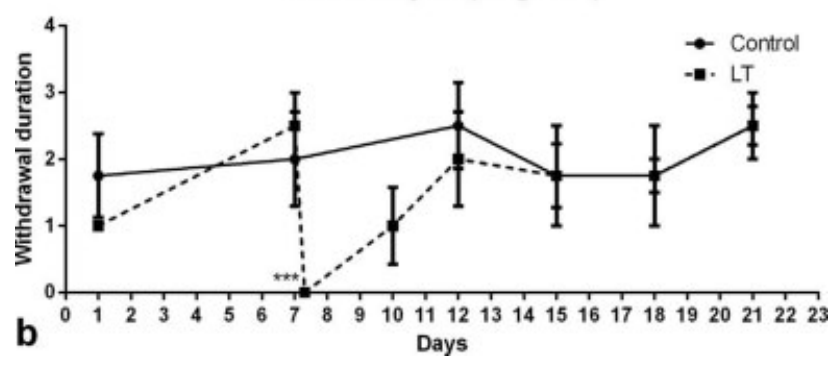

Fig. 5 Cold allodynia. A decrease in sensitivity to the cold stimulus was found within 1 hour post-LT ( $p<0.001$, - Figure 5a). The sensitivity to the cold stimulus returned to the control levels after 5 days post-LT (Day 12) (- Figure $\mathbf{5 b}$ ).

\section{Mechanical Hyperalgesia (Immediately after LT)}

a

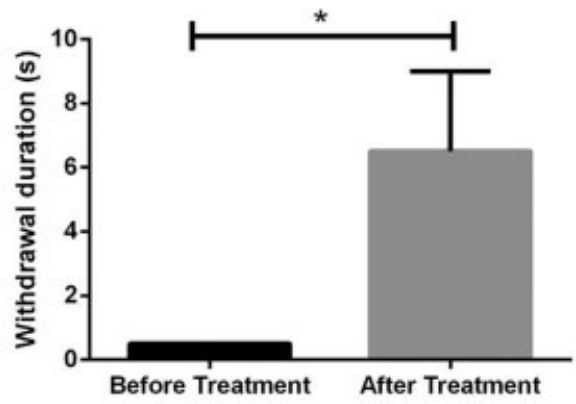

Mechanical Hyperalgesia (long term)

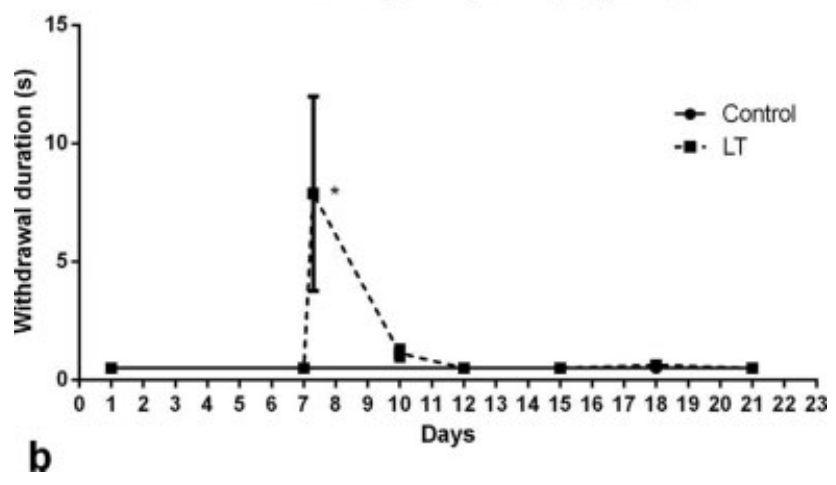

Fig. 6 Mechanical hyperalgesia. A significant decrease in sensitivity to the pin prick stimulus $(p<0.05)$ occurred within 1 hour after LT ( - Figure $6 \mathbf{6}$ ) and returned to the control level after 5 days post-LT on Day 12 (- Figure $6 \mathbf{b}$ ).
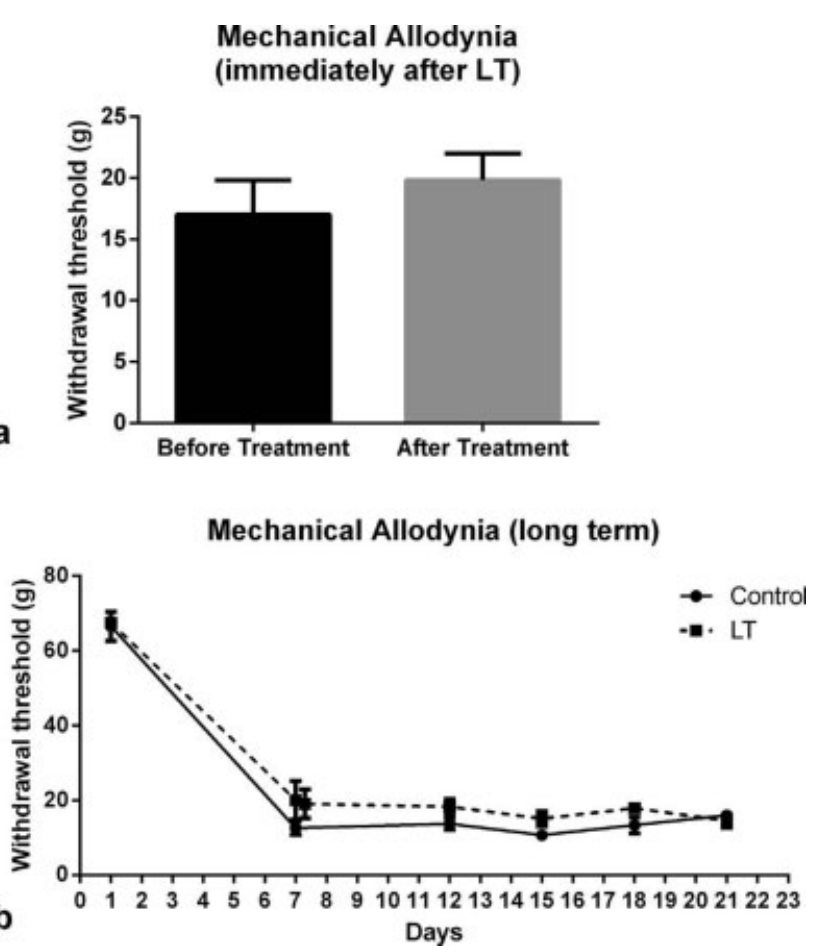

Fig. 7 Mechanical allodynia. No significant difference was found (-Figure $7 a$ and $-7 b$ ). 
examined. This combination of parameters caused a statistically significant decrease in mitochondrial metabolic activities compared with the controls and the cells irradiated with 300 $\mathrm{mW} / \mathrm{cm} 2$ at 60 or 120 s indicating that an irradiance higher that $300 \mathrm{~m} \mathrm{~W} / \mathrm{cm} 2$ may be more effective (data not shown).

Different sizes and types of neurons have differential susceptibility to stimuli that cause axonal varicosity formation. Magdesian et al. (2012) designed experiments to apply gradual nano-scale forces to compress axons of rat hippocampal or DRG neurons in a microfluidic chamber. ${ }^{34}$ They found that the two types of neurons undergo similar morphological changes including varicosity formation but their response differed in intensity and time. The hippocampal axons completely recovered axonal transport when compressed to pressures up to $65 \pm 30$ Pascal(Pa) for 10 min while the DRG axons resisted pressures up to $540 \pm 220 \mathrm{~Pa}$. The authors related the differences in the neuronal response to the composition of the cytoskeletal elements and thus the viscoelastic properties of the axons. They determined that the DRG axons had 7 times more neurofilaments than the hippocampal axons. ${ }^{34}$ Also, neurofilament/microtubule ratios are three times higher in the peripheral nervous system than in the central nervous system. ${ }^{35}$ Furthermore, there are higher numbers of microtubules in unmyelinated axons compared with myelinated axons. ${ }^{36}$ It is important to remember that in our culture model there were no Schwann cells and therefore no myelination of the neurites. Therefore, the response of the neurons to the laser irradiation was not related to the degree of myelination but may be related to differences in the neurofilament/microtubule ratios.

The primary target of the near-infrared light within neurons that results in the cytoskeletal disruption and varicosity formation has not been identified. It has been suggested that the light may be directly absorbed by proteins involved in microtubule stability/instability inducing a conformational change. ${ }^{28}$ Another possibility is that a calcium influx occurs which is followed by the activation of calcium dependent proteases, such as calpain, which cleave and degrade cytoplasmic proteins. ${ }^{37}$

The present study demonstrates that transcutaneous irradiation with an output power of $10 \mathrm{~W}$ delivered $270 \mathrm{~m} \mathrm{~W} / \mathrm{cm} 2$ to the DRG and sciatic nerve and blocked pain and thermal transmission, but did not affect mechanical allodynia which relayed by large myelinated fibers. Laser irradiation of the rat sciatic nerve decreased small and medium sizes fiber somatosensory evoked potentials but did affect fast conducting large fibers. ${ }^{32}$

of clinical relevance is the duration of the effect. As demonstrated, even after 21 days, the laser treated group showed less sensitivity to the heat stimulus compared with control group. For cold allodynia and mechanical hyperalgesia, the duration of the effect was 5 days. In contrast to our results, a study on the assessment of neuropathic pain relief in a chronic constriction injury rat sciatic nerve model reported that laser treatment $(980 \mathrm{~nm}$ wavelength, irradiance on the surface of the skin $=248 \mathrm{~mW} / \mathrm{cm} 2$, irradiation times 16 second at 3 sites) caused an increase in mechanical allodynia and thermal threshold at 7 and 14 days post- surgery. Unlike the present study in which irradiation was done only once, irradiation was done daily for 14 days. ${ }^{38}$

Based on our studies on PBMT and nerve regeneration as well as neuropathic pain and a survey of the literature, we propose two methods that can be used to modulate neuropathic pain based on irradiance levels of near infrared light at the level of the target tissue. The first of these methods uses low irradiances in the range of 10 to $100 \mathrm{~m} \mathrm{~W} / \mathrm{cm} 2$ and causes a decrease in pain response by altering chronic inflammation ${ }^{13}$ and decreasing mechanical allodynia. ${ }^{13,39}$ The mechanisms involved at these irradiance levels are the currently know and accepted mechanisms of PBM. ${ }^{40-42}$ The second method utilizes irradiances in a range from $250 \mathrm{~mW} / \mathrm{cm} 2$ to $1.73 \mathrm{~W} / \mathrm{cm} 2$ which suppress conduction velocity and/or reduce the amplitude of the action potentials ${ }^{16}$ and rapidly block pain transmission as demonstrated in the current pre-clinical animal data and human data. ${ }^{17}$ The mechanism involved at these irradiance levels is the alteration of the neuronal microtubules as discussed above. It is important to note that to transcutaneously deliver these irradiance levels at the target tissue much high levels need to be used at the surface of the skin. We further propose that a combination therapy approach may result in the improved clinical outcomes for treating neuropathic pain. This approach would involve initial use of a high irradiance treatment to block the pain transmission followed by a series of low irradiance treatments along the course of the involved nerve to alter the chronic pathology and inflammation.

\section{Disclosure}

The authors have no financial or other relationship to report that might lead to a conflict of interest.

\section{Acknowledgments}

Part of this study was funded by a CRADA between USUHS and LiteCure LLC. The opinions expressed herein are those of the authors and are not to be construed as reflecting the opinions of the Uniformed Services University of the Health Sciences, the United States Department of Defense, or the United States Army, Navy, or Air Force. This work was presented at ASLMS 2016 and the first author received a travel grant.

\section{References}

1 Tockarshewsky T. Public Comments by the Neuropathy Association at the FDA Anesthetic and Analgesic Drug Products Advisory Committee Meeting. Silver Spring, Maryland: The Neuropathy Association; 2012:3

2 O'Connor AB, Dworkin RH. Treatment of neuropathic pain: an overview of recent guidelines. Am J Med 2009;122(10, Suppl)S22-S32

3 Barbosa RI, da Silva Rodrigues EK, Tamanini G, et al. Effectiveness of low-level laser therapy for patients with carpal tunnel syndrome: design of a randomized single-blinded controlled trial. BMC Musculoskelet Disord 2012;13:248

4 Chow RT, Barnsley L. Systematic review of the literature of lowlevel laser therapy (LLLT) in the management of neck pain. Lasers Surg Med 2005;37(01):46-52

5 Chow RT, Johnson MI, Lopes-Martins RA, Bjordal JM. Efficacy of low-level laser therapy in the management of neck pain: a systematic review and meta-analysis of randomised placebo or activetreatment controlled trials. Lancet 2009;374(9705):1897-1908 
6 Alcântara CC, Gigo-Benato D, Salvini TF, Oliveira AL, Anders JJ, Russo TL. Effect of low-level laser therapy (LLLT) on acute neural recovery and inflammation-related gene expression after crush injury in rat sciatic nerve. Lasers Surg Med 2013;45(04):246-252

7 Byrnes KR, Waynant RW, Ilev IK, et al. Light promotes regeneration and functional recovery and alters the immune response after spinal cord injury. Lasers Surg Med 2005;36(03):171-185

8 Fernandes KP, Alves AN, Nunes FD, et al. Effect of photobiomodulation on expression of IL-1 $\beta$ in skeletal muscle following acute injury. Lasers Med Sci 2013;28(03):1043-1046

9 Peplow PV, Chung TY, Ryan B, Baxter GD. Laser photobiomodulation of gene expression and release of growth factors and cytokines from cells in culture: a review of human and animal studies. Photomed Laser Surg 2011;29(05):285-304

10 von Leden RE, Cooney SJ, Ferrara TM, et al. $808 \mathrm{~nm}$ wavelength light induces a dose-dependent alteration in microglial polarization and resultant microglial induced neurite growth. Lasers Surg Med 2013;45(04):253-263

11 Hsieh YL, Chou LW, Chang PL, Yang CC, Kao MJ, Hong CZ. Low-level laser therapy alleviates neuropathic pain and promotes function recovery in rats with chronic constriction injury: possible involvements in hypoxia-inducible factor $1 \alpha$ (HIF-1 $\alpha$ ). J Comp Neurol 2012;520(13):2903-2916

12 Cidral-Filho FJ, Martins DF, Moré AO, et al. Light-emitting diode therapy induces analgesia and decreases spinal cord and sciatic nerve tumour necrosis factor- $\alpha$ levels after sciatic nerve crush in mice. Eur J Pain 2013;17(08):1193-1204

13 Kobiela Ketz A, Byrnes KR, Grunberg NE, Kasper CE, Osborne L, Pryor B, Tosini NL, Wu X, Anders JJ. Characterization of Macrophage/Microglial Activation and Effect of Photobiomodulation in the Spared Nerve Injury Model ofNeuropathic Pain. Pain medicine (Malden, Mass) 2016

14 Chow RT, Heller GZ, Barnsley L. The effect of $300 \mathrm{~mW}, 830 \mathrm{~nm}$ laser on chronic neck pain: a double-blind, randomized, placebocontrolled study. Pain 2006;124(1-2):201-210

15 Chow RT, David MA, Armati PJ. 830 nm laser irradiation induces varicosity formation, reduces mitochondrial membrane potential and blocks fast axonal flow in small and medium diameter rat dorsal root ganglion neurons: implications for the analgesic effects of $830 \mathrm{~nm}$ laser. J Peripher Nerv Syst 2007;12(01):28-39

16 Chow R, Armati P, Laakso EL, Bjordal JM, Baxter GD. Inhibitory effects of laser irradiation on peripheral mammalian nerves and relevance to analgesic effects: a systematic review. Photomed Laser Surg 2011;29(06):365-381

17 Holanda VM, Chavantes MC, Silva DF, et al. Photobiomodulation of the dorsal root ganglion for the treatment of low back pain: A pilot study. Lasers Surg Med 2016;48(07):653-659

18 Lanzafame RJ, Brandon P, Stadler I. Chapter XII: Wound healing: Experimental and clinical approaches to understanding photobiomodulation and its mechanisms, in Simunovic Z: Lasers ln Medicine And Dentistry. European Medical Laser Association; 20094:163-172

19 Decosterd I, Woolf CJ. Spared nerve injury: an animal model of persistent peripheral neuropathic pain. Pain 2000;87(02):149-158

20 Hargreaves K, Dubner R, Brown F, Flores C, Joris J. A new and sensitive method for measuring thermal nociception in cutaneous hyperalgesia. Pain 1988;32(01):77-88

21 Cobianchi S, Casals-Diaz L, Jaramillo J, Navarro X. Differential effects of activity dependent treatments on axonal regeneration and neuropathic pain after peripheral nerve injury. Exp Neurol 2013;240:157-167

22 Zengin-Toktas Y, Ferrier J, Durif F, Llorca PM, Authier N. Bilateral lesions of the nigrostriatal pathways are associated with chronic mechanical pain hypersensitivity in rats. Neurosci Res 2013;76 (04):261-264

23 Tang-Schomer MD, Johnson VE, Baas PW, Stewart W, Smith DH. Partial interruption of axonal transport due to microtubule breakage accounts for the formation of periodic varicosities after traumatic axonal injury. Exp Neurol 2012;233(01):364-372

24 Burnett MG, Zager EL. Pathophysiology of peripheral nerve injury: a brief review. Neurosurgical focus 2004;16(05):El

25 Kakulas BA. A review of the neuropathology of human spinal cord injury with emphasis on special features. J Spinal Cord Med 1999; 22(02):119-124

26 Saxena S, Caroni P. Mechanisms of axon degeneration: from development to disease. Prog Neurobiol 2007;83(03):174-191

27 Haines JD, Inglese M, Casaccia P. Axonal damage in multiple sclerosis. Mt Sinai J Med 2011;78(02):231-243

28 Tseng CY, Firestein BL. The role of PSD-95 and cypin in morphological changes in dendrites following sublethal NMDA exposure. J Neurosci 2011;31(43):15468-15480

29 Liebert AD, Chow RT, Bicknell BT, Varigos E. Neuroprotective Effects Against POCD by Photobiomodulation: Evidence from Assembly/ Disassembly of the Cytoskeleton. J Exp Neurosci 2016;10:1-19

30 Tanelian DL, Markin VS. Biophysical and functional consequences of receptor-mediated nerve fiber transformation. Biophys J 1997; 72(03):1092-1108

31 Nicolson GL, Smith JR, Poste G. Effects of local anesthetics on cell morphology and membrane-associated cytoskeletal organization in BALB/3T3 cells. J Cell Biol 1976;68(02):395-402

32 Miyamoto Y, Muto E, Mashimo T, Iwane AH, Yoshiya I, Yanagida T. Direct inhibition of microtubule-based kinesin motility by local anesthetics. Biophys J 2000;78(02):940-949

33 Yan W, Chow R, Armati PJ. Inhibitory effects of visible 650-nm and infrared 808-nm laser irradiation on somatosensory and compound muscle action potentials in rat sciatic nerve: implications for laserinduced analgesia. J Peripher Nerv Syst 2011;16(02):130-135

34 Ibáñez CF. Message in a bottle: long-range retrograde signaling in the nervous system. Trends Cell Biol 2007;17(11):519-528

35 Magdesian MH, Sanchez FS, Lopez M, et al. Atomic force microscopy reveals important differences in axonal resistance to injury. Biophys J 2012;103(03):405-414

36 Oblinger MM, Brady ST, McQuarrie IG, Lasek RJ. Cytotypic differences in the protein composition of the axonally transported cytoskeleton in mammalian neurons. J Neurosci 1987;7(02):453-462

37 Maxwell WL. Frontiers in Neuroengineering Development of Concepts in the Pathology ofTraumatic Axonal and Traumatic Brain Injury. In: Kobeissy FH, ed. Brain Neurotrauma: Molecular, Neuropsychological, and Rehabilitation Aspects. Boca Raton (FL): CRC Press/Taylor \& Francis (e); 2015 by Taylor \& Francis Group, LLC. 2015.

38 Lingor P, Koch JC, Tönges L, Bähr M. Axonal degeneration as a therapeutic target in the CNS. Cell Tissue Res 2012;349(01):289-311

39 Masoumipoor M, Jameie SB, Janzadeh A, Nasirinezhad F, Soleimani M, Kerdary M. Effects of 660- and 980-nm low-level laser therapy on neuropathic pain relief following chronic constriction injury in rat sciatic nerve. Lasers Med Sci 2014;29(05):1593-1598

40 Saidu E, Wu X, Alberico S, Pryor B, Smith J, Anders JJ. 980 nm Wavelength Light Decreases Mechanical Allodynia In A Rat Neuropathic Pain Model. Lasers Surg Med 2013;45(25):51

41 Karu TI. Mitochondrial signaling in mammalian cells activated by red and near-IR radiation. Photochem Photobiol 2008;84(05): 1091-1099

$42 \mathrm{Wu} \mathrm{S}$, Cing D. Intracellular signaling cascades following light irradiation. Laser Photonics Rev 2014;8(01):115-130 